\title{
Influence of the electrode gap separation on the pseudospark-sourced electron beam generation
}

\author{
J. Zhao ${ }^{1,2,3, a)}$, H. Yin, ${ }^{3}$ L. Zhang, ${ }^{3}$ G. Shu ${ }^{3}$, W. He $^{3}$, J. Zhang, ${ }^{1,2}$ Q. Zhang, ${ }^{1,2}$ A. D. \\ R. Phelps ${ }^{3}$ and A. W. Cross ${ }^{3}$ \\ ${ }^{1}$ High Voltage Division, School of Electrical Engineering, Xi'an Jiaotong University, Xi'an, 710049, China \\ ${ }^{2}$ State Key Laboratory of Electrical Insulation and Power Equipment, West Xianning Road, 710049, Xi'an, China \\ ${ }^{3}$ Department of Physics, SUPA, University of Strathclyde, Glasgow, G4 ONG, Scotland, United Kingdom
}

Pseudospark-sourced electron beam is a self-focused intense electron beam which can propagate without any external focusing magnetic field. This electron beam can drive a beam-wave interaction directly or after being post-accelerated. It is especially suitable for terahertz $(\mathrm{THz})$ radiation generation due to the ability of a pseudospark discharge to produce small size in the micron range and very high current density and bright electron beams. In this paper, a single-gap pseudospark discharge chamber has been built and tested with several electrode gap separations to explore the dependence of the pseudospark-sourced electron beam current on the discharge voltage and the electrode gap separation. Experimental results show that the beam pulses have similar pulse width and delay time from the distinct drop of the applied voltage for smaller electrode gap separations but longer delay time for the largest gap separation used in the experiment. It has been found that the electron beam only starts to occur when the charging voltage is above a certain value, which is defined as the starting voltage of the electron beam. The starting voltage is different for different electrode gap separations and decreases with increasing electrode gap separation in our pseudospark discharge configuration. The electron beam current increases with the increasing discharge voltage following two tendencies. Under the same discharge voltage, the configuration with the larger electrode gap separation will generate higher electron beam current. When the discharge voltage is higher than $10 \mathrm{kV}$, the beam current generated at the electrode gap separation of $17.0 \mathrm{~mm}$, is much higher than that generated at smaller gap separations. The ionization of the neutral gas in the main gap is inferred to contribute more to the current increase with increasing electrode gap separation.

\section{INTRODUCTION}

A pseudospark discharge is low-pressure, transient discharge which has been recognized as an electron beam source with the highest combined brightness (up to $\left.10^{12} \mathrm{~A} / \mathrm{m}^{2} \mathrm{rad}^{2}\right)$ and current density $\left(>10^{8} \mathrm{~A} / \mathrm{m}^{2}\right)^{1-4}$. The pseudospark-sourced electron beam has been employed in many applications such as material process, free electron laser, x-ray source and microwave devices ${ }^{5-8}$. In recent years,

a) Author to whom correspondence should be addressed. Electronic mail: junping.zhao@qq.com. extensive efforts have been taken to increase the frequency of operation of microwave devices to the $\mathrm{THz}$ region but this often results in the size of the beam-wave interaction region becoming very small in the submillimeter range, which places a much higher requirement on the driving beam current density, these requirements make the pseudospark sourced electron beam a promising candidate $e^{4,9,10}$ to drive $\mathrm{THz}$ radiation sources. Meanwhile, the self-focusing nature of the pseudospark sourced electron beam offers the added benefit of not requiring any external guiding magnetic field, which greatly simplifies the system. Many research works 
have been carried out to study the physics and features related to the pseudospark discharge and electron beam generation. Favre studied the hollow cathode effect in the pseudospark discharge and analyzed the different phases in the pseudospark discharge ${ }^{11}$. Zambra measured the virtual anode velocity under various gas pressures using a capacitive probe $^{12}$. Pitchford analyzed the discharge by numerical simulation and measured energy distribution of the electron beam through a magnetic field deflection method ${ }^{13,14}$. Cetiner simulated the discharge process with different structure and found the influence of the hollow cathode emission and seed electron energy on the beam current ${ }^{15}$, while Gastel studied the influence of the hollow cathode dimensions on the beam current through experiments ${ }^{16}$. Dewald measured the beam energy spectra by a magnetic analyzer and the beam radial profile through fast shutter photography ${ }^{17-19}$. Nistor measured time-resolved electron energy distribution by a self-biased Faraday cup and compared the electron beam energy spectra and ratios between low- and high-energy electrons of single- and multigap configurations by Bremsstrahlung emission and a magnetic analyzer ${ }^{20,21}$. Kumar measured the electron beam pulse and electron beam propagation features under various voltages, as well as the current density distributions with a circular ring detector 22,23 . Yin studied experimentally the features of a single-gap pseudospark discharge, including the discharge voltage, current and electron beam generation under various structures, the propagation and post- acceleration of the pseudospark-sourced electron beam and its application in the generation of microwave radiation ${ }^{24-26}$.

Microwave sources can be driven by the pseudospark produced electron beam directly or post-accelerated and then collimated to the desired shape and size by a collimator mounted before the beam-wave interaction section ${ }^{27}$. But unfortunately the non-monoenergetic nature of this kind of electron beam will reduce the effective density of this type of electron beam. Enhancing the beam current is a practical way of counteracting the tendency for the reduction in the effective density of the electron beam, and can be achieved by studying the influences of the hollow cathode structure on the beam current and by optimizing its parameters ${ }^{15,16,24}$. Very few work has been done to understand the influence of the electrode gap separation on the beam current and the corresponding physics is still unclear although much research has been conducted to study the influence of the gap separation on pseudospark discharges ${ }^{24,28}$.

In this paper, we conduct DC pseudospark discharge experiments to study the dependence of the electron beam current on discharge voltage and electrode gap separation in a pseudospark discharge with the same hollow cathode configuration. Influence of the electrode gap separation on the starting voltage of the electron beam and the peak beam pulse current has been presented and the corresponding primary cause of the features are discussed.

\section{EXPERIMENTAL SETUP}

The DC pseudospark discharge experiments were carried out on the system shown in figure 1. 
The pseudospark discharge chamber under test was applied with a negative high voltage by a Glassman high voltage power supply with output of $0 \sim 100 \mathrm{kV}$ through a protective charging resister with resistance of $2 \mathrm{M} \Omega$. The applied voltage is up to $20 \mathrm{kV}$ to avoid unstable discharge at higher voltage. A capacitor $C_{\text {ext }}$ with capacitance of $\sim 460 \mathrm{pF}$ parallel to the pseudospark gap was used for energy storage. The discharge chamber was evacuated by a vacuum pump and filled with nitrogen gas to the required pressure through a needle valve from a high purity nitrogen gas cylinder with the gas pressure controlled by adjusting a needle valve. The operating pressure of the nitrogen gas was measured using a capacitive nanometer vacuum gauge. The electrode gap separation $d$ can be adjusted by using insulator discs with different thicknesses.

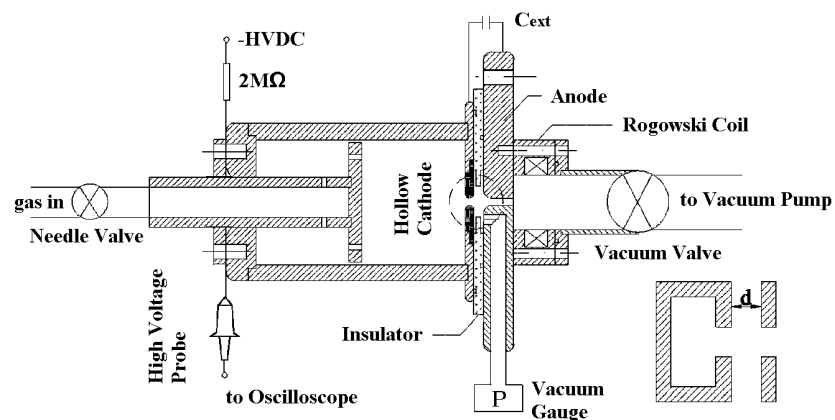

FIG. 1. Experimental setup of the single gap pseudospark discharge.

The discharge voltage and beam current were measured using a high voltage probe (Tektronix P6015A) and a Rogowski coil (homemade self-integrating coil) respectively. The pseudospark configurations as follows were used where both the cathode and anode aperture diameters were $3.0 \mathrm{~mm}$ and gap separations were 4.0, 10.5 and $17.0 \mathrm{~mm}$, abbreviated to $\mathrm{C} 1, \mathrm{C} 2$ and $\mathrm{C} 3$ in the following text, respectively. The aperture edges were rounded with a radius of $1.5 \mathrm{~mm}$ to avoid the undesired emission of electrons during the discharge from the sharp edge to improve the stability of the discharge. The hollow cathode cavity was of a depth and diameter $52.7 \mathrm{~mm}$ and $50.0 \mathrm{~mm}$ respectively. The Rogowski coil was mounted directly onto the anode flange.

\section{RESULTS}

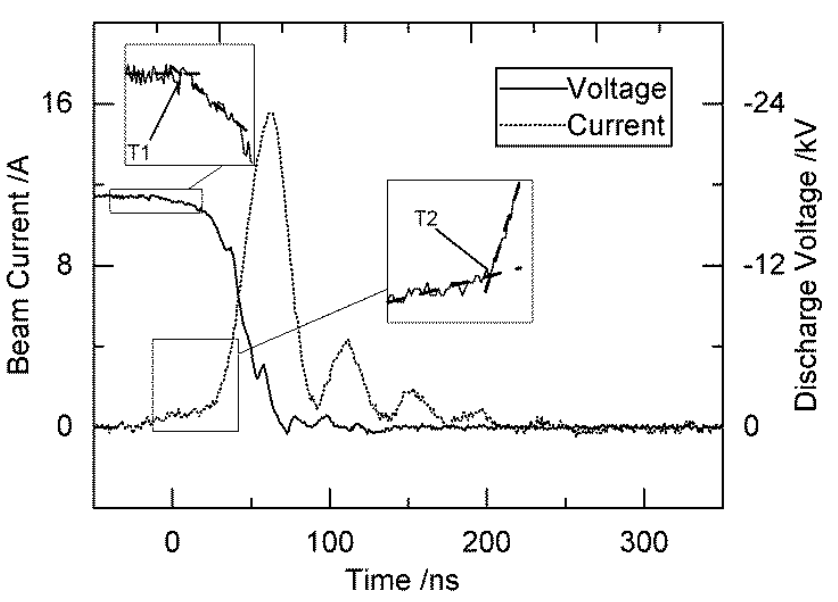

FIG. 2. Typical discharge voltage and beam current waveforms of single-gap pseudospark discharge.

The typical discharge voltage and beam current waveforms are shown in figure 2 . The charging voltage is $17.1 \mathrm{kV}$ and the voltage waveform contains several phases, i.e., unchanged before the discharge and then a slow drop followed by a rapid drop to nearly zero. The waveform of the current contains one main pulse with peak current of $15.5 \mathrm{~A}$ and subsequent smaller pulses. Before the abrupt increase of the main current pulse, there exists a weak current with amplitude of $\sim 0.7 \mathrm{~A}$. This current starts as the voltage starts to drop but with the amplitude still close to the charging voltage, and its propagation along the drift space after the anode will ionize the background gas and generate the plasma channel for self-focusing of the subsequent beam electrons. 
The current pulse peaks under various discharge voltages and electrode gap separations are presented in figure 3. The electrode gap separations in the experiment are 4.0,10.5 and $17.0 \mathrm{~mm}$ respectively as mentioned in section II.

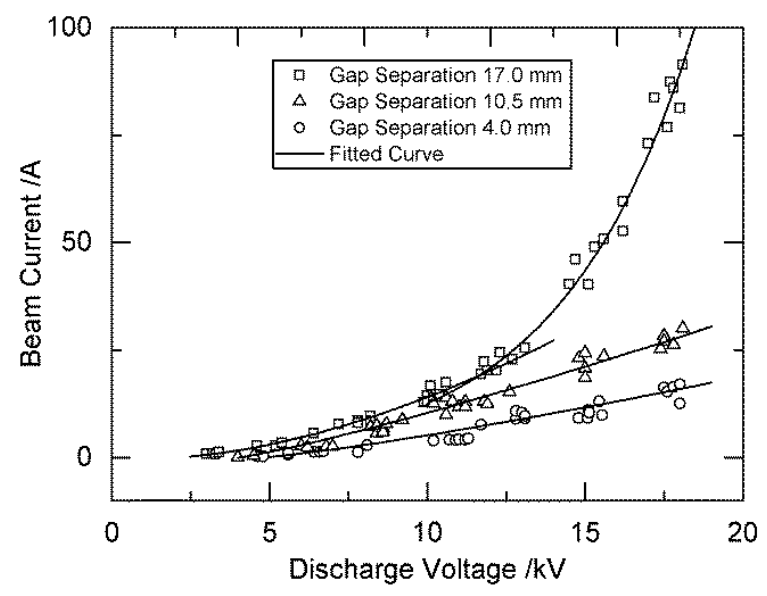

FIG. 3. Beam current peaks with the best-fitted curves of the pseudospark discharge under various discharge voltages and electrode gap separations.

For specific electrode gap separation, the beam current can only be diagnosed when the charging voltage exceeds a certain value, which is defined as the starting voltage of the electron beam. Meanwhile, under the same charging voltage the beam current pulse peak increases as the electrode gap separation increases, as presented in figure 3.

For electrode gap separation of 4.0, 10.5 and $17.0 \mathrm{~mm}$, the starting voltages are around 4.9, 3.7 and $2.4 \mathrm{kV}$ respectively, which will be discussed in Section IV. Discharges below the starting voltage can also take place but without electron beam generation. Consequently, it can be concluded that the higher electron beam can be generated from the pseudospark discharges with larger electrode gap separations. But it is unpractical to enlarge the electrode gap separation indefinitely to obtain higher beam current. In our experiments, a serious unstable discharge occurred when the electrode gap separation was larger than $20 \mathrm{~mm}$. The corresponding gas pressures in the experiments were 14 22, 11 16 and 10 13 Pa for the electrode gap separations of 4.0, 10.5 and $17.0 \mathrm{~mm}$ respectively.

During the voltage dropping, a virtual anode will be formed near the anode and move to the cathode aperture, which will enhance the electrical field near the cathode and cause higher emission of electrons. When the virtual anode penetrates the cathode aperture into the hollow cathode region, dense electron emission will start and the voltage also starts to drop. Then the discharge will transit to the superdense emission stage, and the electron beam current will increase abruptly, the voltage will experience a rapid drop. This is followed by breakdown of the main gap which will cause the end of the main electron beam pulse. Considering the section of the constant charging voltage and slowly dropping part of the voltage waveform as the start time of the virtual anode in cathode region, as marked with $\mathrm{T} 1$ in figure 2, a delay time for the main electron beam pulse can be defined from the start time mentioned above to that corresponding to the transition of the electron beam current from slow to fast increase, which approximately corresponds to the time marked with $\mathrm{T} 2$ in figure 2.

The pulse widths and delays of the electron beam under various discharge voltage and electrode gap separations are presented in figure 4 and figure 5 respectively. With the 
increase of the discharge voltage, the pulse widths of the electron beam rise slightly as well. The pulse widths at the electrode gap separations of both $4.0 \mathrm{~mm}$ and $10.5 \mathrm{~mm}$ are almost the same while the pulse width at the electrode gap separation of $17.0 \mathrm{~mm}$ is about $5 \mathrm{~ns}$ longer than the both on the average.

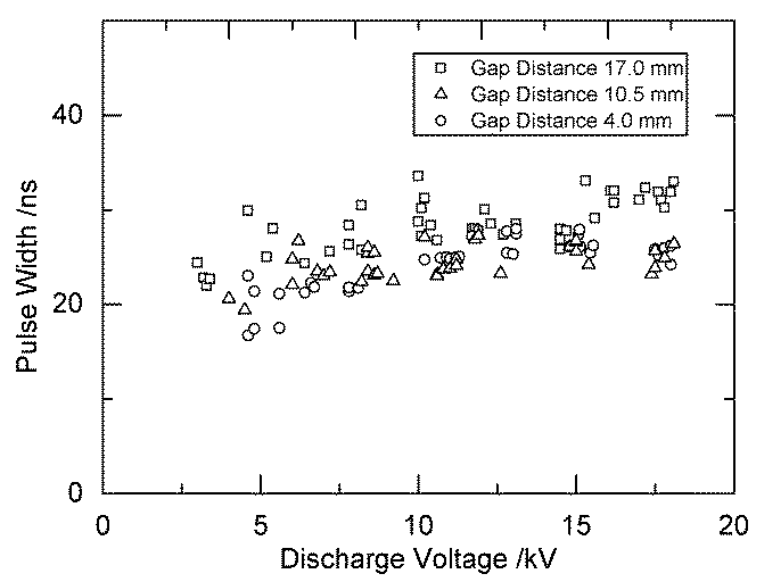

FIG. 4. Pulse widths of the pseudospark-sourced electron beam with various electrode gap separations.

For the pulse delay of the electron beam as defined previously, there exist quite big differences with various electrode gap separations. The pulse delay corresponding to electrode gap separation of $17.0 \mathrm{~mm}$ rises slightly, from nearly $36 \mathrm{~ns}$ to $43 \mathrm{~ns}$, with increasing discharge voltage and is larger than others in most cases except for that near the starting voltage. For the pulse delays corresponding to the electrode gap separations of $10.5 \mathrm{~mm}$ and $4.0 \mathrm{~mm}$, there exists a sharp fall near the respective starting voltage, and then the pulse delays keep to a similar value of $\sim 20$ ns when the discharge voltage is higher than $\sim 8 \mathrm{kV}$.
So the configuration in the experiments has very little influence on the pulse width of the electron beam, while due to the virtual anode kinetics, the pulse delay will be higher with the electrode gap separation larger than $10.0 \mathrm{~mm}$.

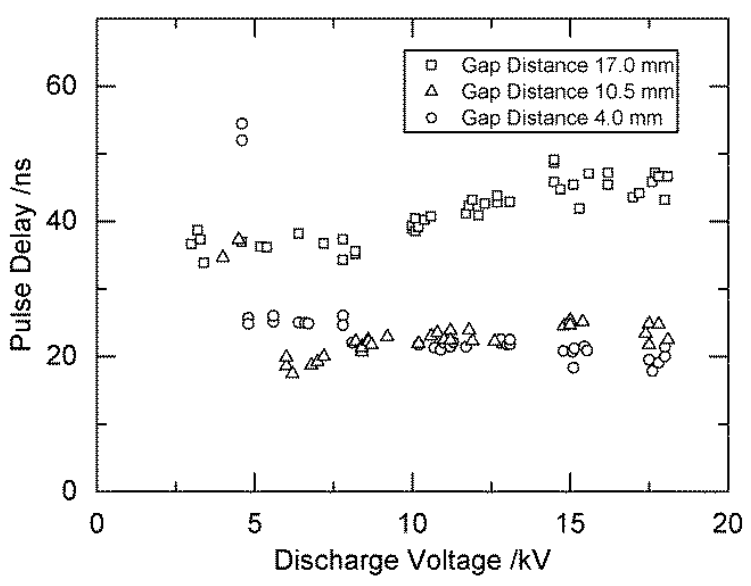

FIG. 5. Pulse delays of the pseudospark-sourced electron beam with various electrode gap separations.

After a virtual anode forms and moves to the cathode, the electron emission of the cathode region and the transition to the breakdown of the discharge are strongly correlated with the activity of the virtual anode. The fact that the pulse widths of the electron beams under various electrode gap separations have similar values indicates that the activities of the virtual anode in the cathode aperture and hollow cathode are similar as long as the cathode structure keeps the same even though the electrode gap separations are quite different.

\section{DISCUSSION}

According to the fact that the electron beam could only emerge when the discharge voltage is above a certain value, the dependence of the maximum beam current on the 
discharge voltage can be assumed to follow the rule in equation (1),

$$
I_{a m p}=a\left(U_{d i s}-b\right)^{c}
$$

where $I_{\mathrm{amp}}$ is the electron beam pulse peak, $U_{\mathrm{dis}}$ is the discharge voltage, $a, b$ and $c$ are constants and $b$ can be considered as the starting voltage of the electron beam current. By fitting the data corresponding to the experimental configurations with the electrode gap separations of $4.0 \mathrm{~mm}$ and $10.5 \mathrm{~mm}$ and part of the data corresponding to that with the electrode gap separation of $17.0 \mathrm{~mm}$ and charging voltage below $13.0 \mathrm{kV}, a, b$ and $c$ can be determined, as represented in table I.

TABLE I. Fitted parameters for equation (1).

\begin{tabular}{cccc}
\hline \hline$d / \mathrm{mm}$ & $a$ & $b$ & $c$ \\
\hline 4.0 & $0.16 \pm 0.02$ & $3.3 \pm 0.3$ & $1.69 \pm 0.44$ \\
10.5 & $0.39 \pm 0.03$ & $2.7 \pm 0.2$ & $1.57 \pm 0.19$ \\
17.0 & $0.22 \pm 0.02$ & $1.9 \pm 0.2$ & $1.91 \pm 0.27$ \\
\hline \hline
\end{tabular}

With the same hollow cathode configuration, the electron beam starting voltages decrease from $3.3 \mathrm{kV}$ to 1.9 $\mathrm{kV}$ with increasing electrode gap separation from $4.0 \mathrm{~mm}$ to $17.0 \mathrm{~mm}$. Under the same discharge voltage for larger separation, the electrical field should be weaker in the main gap region and the hollow cathode region at the initial stage. Supposing it is controlled mainly by the electrical field in the hollow cathode region, the electron emission should be weaker in the configuration with larger gap separation. The multiplication of the electrons in the main gap region should also contribute significantly to the electron beam current, according to the experimental results that the configurations with larger separations have lower starting voltage and higher beam current under the same discharge voltage, as shown in figure 3 and table I.

As shown in figure 3, with the gap distance of $17.0 \mathrm{~mm}$, the dependence of the electron beam pulse peak on the discharge voltage shows a different tendency when the discharge voltage is higher than $\sim 10 \mathrm{kV}$. The beam pulse peak increases far more rapidly with the increasing discharge voltage than those in other cases. Suppose the beam current peak and the discharge voltage following the rule in equation (2),

$$
I_{a m p}=f^{\left(U_{d i s}-g\right)}+h
$$

By fitting the corresponding experimental data, $f, g$, and $h$ can be determined as $1.26 \pm 0.08,-1.19 \pm 0.06$ and $-1.28 \pm 0.12$. The curves corresponding to the fitted parameters are presented in figure 3 .

The delay time shown in figure 5 corresponds to the time from the formation of the virtual anode to its penetration into the hollow cathode aperture, that is the virtual anode travelling time across the space between the cathode and anode. According to Lucas's model, the velocity of the virtual anode towards the cathode is critically dependent on the emission rate of the cathode region ${ }^{29}$, while in Zambra's and Choi's study, the velocity of the virtual anode can be significantly influenced by the gas pressure ${ }^{12,30}$. In our experiment, with the particular gap separation, as the discharge voltage increases, the gas pressures decreases, from 22, 16 and $13 \mathrm{~Pa}$ to 14,11 and $10 \mathrm{~Pa}$ for the gap separations of 4.0, 10.5 and $17.0 \mathrm{~mm}$ respectively. According to Zambra's and Choi's work, the delay time should be larger 
with higher discharge voltage because of the lower velocity of the virtual anode corresponding to the lower gas pressure, while the experimental data shows that the delay times for particular gap separations keep almost the same. It can be inferred that the higher electron emission corresponds to higher discharge voltage and speed of the virtual anode formation and movement towards the cathode, which counteracts the lag caused by the decreased pressure, according to Lucas's model. Although the distance from the anode to the cathode increases more than twice when the gap separation is changed from $4.0 \mathrm{~mm}$ to $10.5 \mathrm{~mm}$, the delay is approximately equal, as presented in figure 5. It can also be inferred that the higher electron emission corresponding to the larger separation plays an important role in the counteraction of the delay. For the largest separation in our experiment, the gas pressure and gap distance dominate the delay time, which is $\sim 20$ ns longer than with other configurations but still keeps approximately equal with increasing discharge voltage, as presented in figure 5.

The detailed physics of the pseudospark discharge is still not completely understood. It is generally agreed that the intense electron emission starts at the stage of the hollow cathode discharge. When the virtual anode arrives at the cathode and penetrates into the hollow cathode through the cathode aperture, intense electron emission of the cathode region starts and the beam current starts to increase rapidly. Favre considered that the collapse of the sheath near the hollow cathode surface may cause the subsequent breakdown of the main gap ${ }^{11}$. While Korolev considered that the microexplosion of the cathode surface finally causes the breakdown of the main gap during the superdense glow discharge stage ${ }^{31}$. Despite the specific description of the process, the transition time from the virtual anode reaching the cathode region to the breakdown taking place should be mainly controlled by the hollow cathode structure and the applied electrical field, so the duration of the transition should be similar for some configurations with the same hollow cathode structure and cathode aperture as well as the same circuit configuration, that is to say the electron beam pulse width should also be similar.

According to the experimental measurements of the delay time and pulse width, it can be inferred that the emission in the cathode region should be similar for different gap configurations. Those electrons that left the cathode region, will be driven towards the anode by the applied electric field and the electron impact ionization may occur. The mean number of the ionization during this period can be roughly estimated through equation (3),

$$
\bar{n}_{i}=n \bar{\sigma}_{i} d
$$

where $\bar{n}_{i}, n, \bar{\sigma}_{i}$ and $d$ are the mean number of electron impact ionization, the neutral gas density, the mean cross section of electron impact ionization and the main gap separation respectively. For nitrogen molecule, the maximum electron impact ionization cross section is around $2.5 \times 10^{-16} \mathrm{~cm}^{2}$. For the separations of $4.0 \mathrm{~mm}, 10.5 \mathrm{~mm}$ and $17.0 \mathrm{~mm}$, the corresponding maximum mean numbers of the electron impact ionization can be $0.23-0.53,0.69-1.01$ and $0.92-1.33$, with the corresponding gas pressures respectively. This 
means that, for an electron moving from the cathode to the anode, the possibility of the electron impact ionization increases with the increasing separation although the corresponding nitrogen molecule density decreases. This can be the primary reason that the electron beam current is higher with larger electrode gap separation, as presented in figure 3 .

According to the electron beam energy spectra obtained by

Edward and Nistor, most of the electrons in the beam are of low-energy ${ }^{19,21}$ and the energy spread increases over the time due to the increase of the low-energy electrons ${ }^{20}$. The increase of the low-energy electrons is due to the energy loss resulting from the electron collision during the beam transport and, from another perspective, this can be a proof of the electron impact ionization in the main gap.

\section{CONCLUSION}

For the pseudospark-sourced electron beam, we studied the beam current generation under various electrode gap separations with the same hollow cathode configuration. For smaller electrode gap separations, the beam pulses are of similar pulse width with similar delay from the distinct drop of the applied voltage. It has larger delay for the largest electrode gap separation. From the experimental data, it has been found that the start voltage of the electron beam is different with various electrode gap separation and decreases with increasing electrode gap separation in our pseudospark cavity configuration. The electron beam current increases with the increasing discharge voltage following two tendencies. The configuration with larger electrode gap separation will generate an electron beam with higher current under same discharge voltage. Especially when the discharge voltage is higher than $10 \mathrm{kV}$, the beam pulse peak with the gap separation of $17.0 \mathrm{~mm}$ is much higher than that with the other two smaller gap separations. The ionization of the neutral gas in the main gap is inferred to contribute more to the current increase with increasing electrode gap separation.

\section{ACKNOWLEDGMENTS}

The authors would like to thank David Barclay of the Atoms, Beams and Plasmas group at the Department of Physics, University of Strathclyde UK for constructing the components of the pseudospark cathode.

\section{REFERENCES}

${ }^{1}$ Y. Huang, M. C. Wang, and Z. J. Wang, Chinese Science Bulletin 43, 260 (1998).

${ }^{2}$ W. Benker, J. Christiansen, K. Frank, H. Gundel, W. Hartmann, T. Redel, and M. Stetter, Plasma Science, IEEE Transactions on 17, 754 (1989).

3 J. Christiansen and C. Schultheiss, Zeitschrift für Physik A Atoms and Nuclei 290, 35 (1979).

${ }^{4}$ W. He, L. Zhang, D. Bowes, H. Yin, K. Ronald, A. D. R. Phelps, and A. W. Cross, Applied Physics Letters 107 (2015).

5 S. V. Lebedev, M. Machida, S. A. Moshkalyov, and D. O. Campos, Ieee Transactions on Plasma Science 25, 754 (1997).

6 J. B. Zhu, M. C. Wang, Z. J. Wang, and J. K. Lee, Optical Engineering 35, 498 (1996).

${ }^{7}$ C. S. Wong, H. J. Woo, and S. L. Yap, Laser and Particle Beams 25, 497 (2007).

${ }^{8}$ A. W. Cross, H. B. Yin, D. Bowes, W. L. He, K. Ronald, and A. D. R. Phelps, New Developments in Nonlinear Plasma Physics 1188, 380 (2009).

9 T. Schuhmann, J. Protz, D. Fields, H. Yin, A. Cross, W. He, D. Bowes, K. Ronald, and A. Phelps, Millimetre Wave and Terahertz Sensors and Technology Iii 7837 (2010).

${ }^{10}$ Y. Yin, W. L. He, L. Zhang, H. B. Yin, C. W. Robertson, and A. W. Cross, Ieee Transactions on Electron Devices 63, 512 (2016).

${ }^{11}$ M. Favre, P. Choi, H. Chuaqui, Y. Kaufman, J. Moreno, E. Wyndham, and M. Zambra, IEEE Transactions on Plasma Science 23, 212 (1995).

${ }^{12}$ M. Zambra, J. Moreno, J. Inostroza, and J. C. Araneda, IEEE Transactions on Plasma Science 32, 221 (2004). 
${ }^{13}$ L. C. Pitchford, Journal of Applied Physics 75, 7227 (1994).

${ }^{14}$ L. Pitchford, J. P. Boeuf, V. Puech, R. Liou, and M. Gundersen, Proceedings of the 1993 Particle Accelerator Conference, Vols 1-5, 3069 (1993).

${ }^{15}$ S. O. Cetiner, P. Stoltz, P. Messmer, and J.-L. Cambier, Journal of Applied Physics 103, 023304 (2008).

${ }^{16} \mathrm{M}$. Gastel, H. Hillmann, F. Muller, and J. Westheide, Ieee Transactions on Plasma Science 23, 248 (1995).

${ }^{17}$ E. Dewald, K. Frank, D. H. H. Hoffmann, M. Ganciu, N. B. Mandache, M. Nistor, A. M. Pointu, and I. I. Popescu, Nuclear Instruments \& Methods in Physics Research Section a-Accelerators Spectrometers Detectors and Associated Equipment 415, 614 (1998).

${ }^{18}$ E. Dewald, K. Frank, D. H. H. Hoffmann, R. Stark, M. Ganciu, B. N. Mandache, M. G. Nistor, A. M. Pointu, and I. I. Popescu, IEEE Transactions on Plasma Science 25, 272 (1997).

${ }^{19}$ E. Dewald, M. Ganciu, B. N. Mandache, G. S. Musa, M. G. Nistor, A. M. Pointu, I. I. Popescu, K. Frank, D. H. H. Hoffmann, and R. Stark, IEEE Transactions on Plasma Science 25, 279 (1997).

${ }^{20} \mathrm{M}$. Nistor and N. Mandache, J. Optoelectron. Adv. Mater 7, 1619 (2005).

${ }^{21}$ M. Nistor, P. Charles, M. Ganciu, M. Lamoureux, N. B. Mandache, and A. M. Pointu, Plasma Sources Science and Technology 11, 183 (2002).

${ }^{22}$ N. Kumar, D. K. Verma, J. Prajapati, M. Kumar, B. L. Meena, M. S. Tyagi, V. Srivastava, and U. N. Pal, International Symposium on Vacuum Science and Technology and Its Application for Accelerators (Ivs 2012) 390 (2012).

${ }^{23}$ N. Kumar, U. N. Pal, D. K. Verma, J. Prajapati, M. Kumar, B. L. Meena, M. S. Tyagi, and V. Srivastava, Journal of Infrared Millimeter and Terahertz Waves 32, 1415 (2011).

${ }^{24}$ H. Yin, W. He, A. W. Cross, A. D. R. Phelps, and K. Ronald, Journal of Applied Physics 90, 3212 (2001).

${ }^{25}$ H. Yin, A. W. Cross, A. D. R. Phelps, D. Zhu, W. He, and K. Ronald, Journal of Applied Physics 91, 5419 (2002).

${ }^{26}$ H. Yin, A. W. Cross, D. Bowes, W. He, A. D. R. Phelps, K. Ronald, D. Li, J. Zhou, X. Chen, J. Protz, M. Verdiel, M. Reynolds, and T. Schuhmann, 2009 34th International Conference on Infrared, Millimeter, and Terahertz Waves, Vols 1 and 2, 596 (2009).

${ }^{27}$ H. Yin, A. W. Cross, W. He, A. D. R. Phelps, and K. Ronald, Ieee Transactions on Plasma Science 32, 233 (2004).

${ }^{28}$ J. Zhang, J. Zhao, and Q. Zhang, IEEE Transactions on Plasma Science 42, 2037 (2014).

${ }^{29}$ L. James, Thesis (Ph.D.) Thesis, 1961.

${ }^{30} \mathrm{P}$. Choi, H. Chuaqui, M. Favre, and V. Colas, Plasma Science, IEEE Transactions on 23, 221 (1995).

${ }^{31}$ Y. D. Korolev and K. Frank, IEEE Transactions on Plasma Science 27, 1525 (1999). 